\title{
1. Introduction to the governance of home care
}

This chapter introduces home care as a complicated set of activities that transcend a number of boundaries, especially between public and private, formal and informal, and the micro and the macro levels. We discuss the importance of defining care by its location and provide an overview of the different locations that are used to define 'home', as well as the dynamics between different home locations. This chapter also introduces the concept of 'governance' as applied to home care, and introduces the close relationship between the public/private and the formal and informal sectors. ${ }^{1}$ It demonstrates that the governance of home care has become highly politicised because of demographic developments relating to ageing populations, societal developments relating to individualisation and changing gender cultures, including changes in the traditional role of families as care givers, and economic developments relating to heightened resource constraints across all policy areas.

This chapter also explains our choice of countries for analysis and offers an overview of the relevant cultural and institutional contexts of home care in each country, which are fleshed out in Chapter $3 .^{2}$ Finally we introduce the issue of convergence. At the theoretical level there is currently considerable debate between those who argue that there is a convergence of policy across countries and others who contend that despite convergence, in some arenas there remains considerable divergence due to cultural and structural variation. These latter observers point to the embeddedness of policies in countryspecific contexts. Home care governance across these countries serves as an excellent test of the convergence hypothesis.

\section{DEFINING HOME CARE}

The user groups of home care are diverse and reflect the support required at different stages of the life span, ranging from severely ill infants to people at the end of their life. Other beneficiaries of home care include people with mental illness and handicap, physical disability, drug-related disorders and progressive illness (Means et al., 2003). Each of these groups raises a different 
set of issues and problems. Thus to provide a workable focus, this book deals exclusively with home care for older people. Already older people constitute by far the largest user group of home care, a figure that will increase significantly in the light of the ageing of societies, discussed in more detail below.

We use Tester's definition of home care as 'any type of care and support offered to older people in their homes, whether ordinary or specialised settings, by formal and informal carers' (1996: 76). Home care thus encompasses a wide range of tasks and activities, and cuts across the boundaries between health and social care and between formal and informal care. It includes basic mobility and self-care, medical and nursing care to help with physical and mental health problems, help in daily living tasks, counselling and emotional support to promote well-being; as well as other social, educational and leisure activities (Tester, 1996). Home care usually involves nurses rather than doctors, as well as home helps and most importantly women as informal carers. Indeed, unpaid carers deliver most home care, and it is estimated that formal care comprises only one-fifth of the total help older people receive (Jacobzone, 1999). The diversity of care subsumed under home care reflects the disparate yet interlocking needs of people who require longterm care. Diversity makes such care interesting but also difficult to define, analyse and compare. Moreover, care services are often locally specific and even tailored to particular individuals, and it is difficult to identify the typical case let alone to generalise.

Home care, then, is a highly complex activity that includes not only a wide range of different tasks but also transcends the distinction between formal services and informal relationships. The former links home care governance to the dynamics of policy making and different approaches to governing, whereas the latter links home care governance to the wider family and gender structures in society. Informal care in turn can be delivered by trained professionals, untrained and often poorly paid helpers, family or friends. Home care also crosses the boundaries between the domestic and public life and the macro and micro levels. The boundary between public and private which is evident in more traditional policy areas is blurred in home care. Although some of these services might be provided formally through an array of public and private organisational structures, much home care is informal and thus outside the purview of formal controls, often provided by friends or relatives without any regularised rules of guidance. Home care also obscures the distinction between the macro and micro levels and introduces a tension between hierarchical and individualistic approaches. Because it can be so multifaceted and decentralised, home care requires analysis at both levels simultaneously.

Chapter 3 examines in more detail the interface between formal and 
informal care and the underpinning cultural expectations about the role of women in care giving. This linkage is important because, as acknowledged by recent policy initiatives in many countries, most home care continues to be provided by unpaid carers, primarily women. However, under the banner of greater welfare mix, the objectives of reforms are to integrate carers more explicitly into formal care arrangements and to support carers through payments and related benefits.

Likewise, the line between home and institutional care remains an ambiguous one. In the US and to a lesser extent other countries, assisted-care living facilities have been initiated that combine the two concepts. Usually the facility has three levels of care depending on the health and mobility of the older person. Homes and apartments in the independent care section allow those who need particular care services to get them. As they need more extensive care they move into a room or apartment that provides a more intensive care environment. And finally, they can move to a nursing home facility located in the same complex. In the US, these care facilities are a mix of for-profit (usually more high-end facilities) and non-profit (often church or charity based). The point here, though, is that while the literature conceptually distinguishes home from institutional care, in reality this distinction is blurred. With this caveat in mind, however, our analysis will focus on the location of care in the home setting.

\section{FRAMEWORK FOR STUDYING THE GOVERNANCE OF HOME CARE}

In order to structure our analysis of governing home care in a helpful manner we have constructed the framework in Figure 1.1. Because home care crosses many traditional boundaries in public policy analysis (formal/informal, public/ private, macro/micro) this book addresses the concept of governance instead of the more traditionally studied public policy. This allows us to account for the complexity of home care where public policy is but one element. We contend that to understand home care governance we must examine all the relevant institutions and ideas as well as actors. In terms of institutions we shall analyse social institutions (especially relating to gender arrangements), political institutions (including sector-specific institutional arrangements, but also broader institutional arrangements such as the welfare state and the political system), and the opportunities and constraints institutions make in relation to governance options. Finally, in terms of ideas we analyse culturally embedded traditions, beliefs and attitudes relating to home care and the changing nature of ideas (as reflected in 'critical instances' that introduce new ideas and help to account for changing governing arrangements). 
Context of home care governance

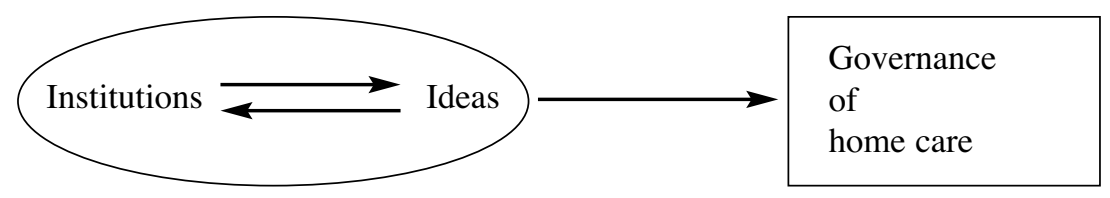

Figure 1.1 Framework for analysing the governance of home care

This analytical framework aims to map out and differentiate among the many ways in which the governance of home care comes about. It is used to map out home care and its organisation and to account for differences and similarities across countries by analysing relevant ideas and institutions and the interplay between them. There are many processes (understood as interactions between institutions and ideas) which shape the governance of home care but which do not go through the formal policy process. This infers that a significant degree of governing goes on beyond government.

Where appropriate, our analysis also looks at explicit processes of policy making. This involves examining the many actors involved, both veto/status quo actors and policy entrepreneurs. The first type is likely to try to keep home care off the political agenda while the second type can be crucial in putting home care on the political agenda. Moreover, these actors can come from within government or from outside. It is also important to note that policy here has two dimensions and entails both specific home care policies and other policies that impact on home care. Because home care transcends many areas, the second dimension is of special relevance in the absence of a dedicated home care policy. In fact we shall see that direct governance through specific policies has been the exception.

It should be emphasised here that despite the directional arrow in our model, it is not meant to describe causal relations but instead characterises a loose and flexible configuration of factors that shape the governance of home care. With many factors operating in each country, the effect of individual factors depends as well on the interplay among them, thus obscuring the formulation of any precise predictions. Despite this caveat, we feel that this framework is valuable in making sense of home care governance across our countries.

As noted earlier, our focus here is on a comprehensive notion of governance of home care rather than public policy in the more restrictive sense. We argue that to understand home care one must analyse different logics of governance (state, associations, market, family) as well as different instruments of governance (delivery, funding and rules) that taken together gives us a fourby-three table that will be used for comparison of the countries (Table 1.1). This more inclusive, and we argue accurate, view of the governance of home care demonstrates that in order to understand differences and similarities of 
home care across countries we must be aware of the activity not only by the state, but also by associations, the marketplace and the family. Moreover, the instruments of governance will vary not only by locale but also across time, thus explicating the dynamics of home care governance. Chapter 2 will provide more details on the logics and instruments of governance drawn from the literatures of social care and public policy and fill in the blank spaces in Table 1.1.

Table 1.1 Logics and instruments of home care governance (1)

Logics of governance Instruments of governance

Funding Delivery Rules

State

Associations

Market

Family

Governance, then, is an umbrella concept covering a broad range of mechanisms for creating order among different actors in the public sector. Governance moves beyond the purely private to collective action. 'Among the issues which governance invites one to analyse are the relationships among local, regional and national levels, the role of the state and its relationship to civil society, the (re)positioning of different interest groups and the framing, orientation and implementation of policies' (Daly, 2003: 115). Moreover, the locale and exercise of power are central to governance and it is especially attuned to the changing set of arrangements between the state and the other logics. As noted by Jessop (1999), the state is more than a political agent; it is also the bearer of certain types of social packages. This latter is a role that is changing as the welfare state shrinks and globalisation intensifies.

As suggested in the discussion of the analytical framework above, there are two routes to governance of home care, a direct route and an indirect one where the policy process acts as a filter. Although we emphasise a comprehensive analysis of the varied logics, the core intervening variable in home care governance is public policy defined here as an action (or nonaction) taken by the government or on behalf of it (Heywood, 1997). It is a goal-driven course of action designed to promote, maintain or prevent a particular state of affairs. While other organisations such as medical associations and care societies often make decisions that affect many individuals as well as the home care system, only the government has the 
legitimate authority to make decisions that are binding and carried out in the name of the people as a whole.

Home care policy is defined here as those courses of action or non-action proposed or taken by a government that impact on the financing, delivery or rule of home care services. However, while attention is understandably focused on the government, we argue that inclusion of the other logics of governance, including the actions by private and voluntary organisations, the market and the family that impact on home care governance are crucial in crossing the boundaries between public and private dimensions. In some countries, state non-action in the home care arena has been a more common approach than action. Also critical is the fact that although home care conceptually can be distinguished from other areas of public policy, in reality it is highly interrelated with social and economic policy in general. Thus home care policy cannot be understood in isolation from social welfare, health, unemployment, poverty, housing and general economic policies. Therefore where relevant we bring these other policy areas into our analysis.

\section{COMPARATIVE HOME CARE GOVERNANCE}

In order to elucidate the similarities and differences in approach among various countries' efforts to resolve similar problems and govern home care, this book places home care governance in a comparative context. Although it is dangerous to apply uncritically policies that work in one county to those in another, comparative policy analysis is useful in expanding policy options and demonstrating the experiences of a wide range of applications. Antonnen et al. (2003a), for instance, conclude that 'current patterns of globalisation in politics, economics and culture and the growing movements of people across borders have raised the significance of comparative social research' (2003: 7). Ovretveit (1998) adds that travel and new information systems are making it both easier and more necessary to understand cultural and national differences. Harrop agrees that 'by examining policies comparatively, we can discover how countries vary in the policies they adopt, gain insight into why these differences exist, and identify some of the conditions under which policies succeed or fail' (1992: 3). Ham (1997: 50) contends that an examination of international experience is illuminating in demonstrating both the difficulties faced by and the wide range of approaches available to policy makers, both public and private.

Comparative studies, then, can give us cross-cultural insights as to governance of home care under a wide variety of institutional and value contexts. For instance, which countries have taken a pro-active approach by making and implementing specific home care policy and which have deferred 
governance to associations, the market or the family? Given the complexity of governing home care, we argue that only comparative studies can generate the evidence necessary to consider the full array of options.

Comparative analysis can also illustrate the commonalities of problems and variables across countries. Although to some extent home care governance is unique to each nation, one cannot ignore the globalisation of problems and potential solutions. These global forces are seen by some as supporting the convergence theory, which argues that as countries industrialise they tend to converge towards the same policy mix (Bennett, 1991). Bolstered by globalisation, the convergence thesis suggests that home care governance across disparate country environments will have a tendency to become more similar over time as they develop economically. Gibson and Means (2000), for example, argue that recent restructuring has led to convergence of the longterm care regimes of Australia and the UK, despite quite dissimilar goals and strategic emphases.

Critics of convergence theories, however, argue that they oversimplify the process of development and underestimate significant divergence across countries (Guillén, 2001, Howlett and Ramesh, 2003: 107). They argue that convergence downplays the importance of country-specific factors other than economic development and that most studies that find evidence of convergence do not find it applicable across the board, thus allowing for divergence in other areas. We also surmise that because home care deals with less standardised, more personalised services often at the local level, any forces of convergence apparent at national levels are likely to be marginalised or at least less consistent than in more formal policy areas. At the least, home care would seem more resistant to convergence than, for instance, health care. Although this book is not designed to test the convergence theory directly, its current prominence in the literature dictates that where appropriate its assumptions be addressed in the following chapters and that its explanatory power across these countries be explored more fully.

\section{COUNTRIES SELECTED FOR ANALYSIS}

In order to provide a useful cross-country analysis of home care governance, we selected nine countries for primary coverage. These countries were chosen to give the reader systematic exposure to the full range of institutional and cultural contexts. Although they are all developed countries they differ widely in terms of institutions, ideas and associated actor combinations, and as such they represent a wide spectrum of political, cultural and economic environments for governing home care. ${ }^{3}$ The group of case countries includes some such as Germany, Sweden, the Netherlands, the UK and the US that are 
often included in comparative studies of Western nations, and others such as Estonia, Italy, Japan and New Zealand that are seldom included. ${ }^{4}$ Individually, each of the countries has unique contributions to make to the study of home care governance. In combination, these countries serve as a constructive sample upon which to analyse the dynamics of home care governance in the twenty-first century.

Our model suggests that home care governance in any country at a particular point in time is the product of a multitude of factors, including the intrinsic social, cultural and political fabric of a country, which encompass social values and structures, political institutions and traditions, the legal system, and characteristics of the health and social care communities. These institutions and ideas (and actors) can influence home care governance directly or be shaped and filtered through the policy process. For that portion of home care governance impacted on by the policy process it is crucial to understand the characteristics of that process in each country (Anttonen et al., 2003a: 174). For instance policy-making authority can be highly centralised or widely dispersed across multiple levels. Moreover in some countries, unions and/or corporate structures are strong factors in determining social policy and might in effect have a veto power over proposed policy changes made by the government.

\section{Contrasting Political Systems}

Although the political systems of no two countries are identical and each nation has a unique combination of formal and informal structures, the characteristics of political systems can be categorised along several overlapping dimensions (Lijphart, 1999). The major distinguishing factor is the extent to which political power is concentrated or dispersed. As a rule, unitary systems concentrate political authority in a central government while federal systems constitutionally divide powers between the central government and states, provinces or other subnational levels. Although the central government in a unitary system might choose to delegate specific administrative functions and responsibilities to lower units, final authority rests at the national level. In contrast, states in a federal system have constitutionally-based powers that very often include health and social welfare policy. Most European countries have unitary systems while Germany, the Netherlands, and the US are examples of the federal model.

Another institutional configuration that relates directly to the continuum of centralisation is the distribution of power within each level of government. On the one hand many democracies, such as New Zealand and the UK, concentrate power in parliament where the distinction between executive and legislative power is obscured or virtually non-existent. In stark contrast to 
other countries examined here is the US, with its deliberate constitutional separation of powers among a separately elected president, two houses of Congress and a relatively active judicial system. Despite a great deal of variation in the diffusion of policy-making authority in some parliamentary systems as compared to the US, they all display considerably more concentrated bases of power. Figure 1.2 presents a rough distribution of our case countries in terms of centralisation of institutional power along these dimensions. This distribution corresponds closely to the findings of Lijphart (1999: 189), who classifies Germany and the US as 'federal and decentralised'; the Netherlands as 'semi-federal'; Japan and Sweden as 'unitary and decentralised'; and Italy, New Zealand, Estonia and the UK as 'unitary and centralised'.

$\begin{array}{cccc}\text { Estonia } & \text { Italy } & \text { Japan } & \text { Germany } \\ \text { New Zealand } & \text { UK } & \text { Sweden } & \text { Netherlands }\end{array}$

Concentrated

Fragmented

\section{Figure 1.2 Institutional power in political systems}

The implications of these formal government types for home care governance are likely to be significant. Where power is highly centralised, the government has the formal capacity to make more rapid and comprehensive policy changes. In contrast, the more fragmented the political authority the greater the probability of deadlock and inaction or at best more incremental change. Moreover, in fragmented political systems where competing political parties are able to control particular institutions, there is more likely to be a divided government where one party controls one or several branches or levels and the other party controls others. Although this might contribute towards a more deliberative policy-making process, it can also degenerate into stagnation and gridlock as has been commonplace in the US. Conversely, it might be expected that a highly centralised system such as the UK or Italy produces a policy arena characterised by more frequent and inclusive changes such as major restructuring, often to the detriment of programme stability.

The most obvious dimension of the political context consists of the formal institutions that have been created for making public policy decisions and which define the distribution of power and the relationships among the political players. As such they give distinct advantages or disadvantages to various groups in society. Equally important to understanding governance are the informal practices and structures that have evolved within a particular formal institutional framework. These traditions and rules of the game define a different political logic in each country. They, too, are critical to the 
environment within which interest groups, political parties, bureaucrats and individual politicians vie for influence over policy. Together, these formal and informal political institutions shape how politics is conducted and create a strategic context for political conflict within the policy arena. 'Political factors help to determine whether a problem is defined as a public policy that requires action, they shape the way in which the problem is defined, and they intervene in the resolution of that problem' (Immergut, 1992: 10). Although institutional variables are not the only ones that matter, variation in institutional conditions across countries yields different opportunities for the actors involved in policy making in each country (Timmermans, 2001: 311).

\section{The Social, Cultural and Historical Context of Home Care}

Each country also brings to home care governance a unique combination of social institutions, and historical and cultural factors that are crucial in explaining its proclivities and characteristics. For instance, political culture is the complex of beliefs, values and attitudes held by the public concerning the proper role of government. To what extent is home care a public as opposed to a private good and, if a public good, on what grounds should it be distributed to individuals? What is the perceived role of the state as opposed to the citizen? In home care especially these cultural differences have broad implications for gender because of the fact that women traditionally have been the primary care givers to the elderly. Likewise, any differences in how societies define the roles of women are likely to impact directly on home care. In order to provide a basis for more in-depth comparison throughout later chapters, we now look briefly at the key defining cultural characteristics that might influence home care, gendered ways in which the individual in society relates to the whole. Table 1.2 shows a rough distribution of these countries as to whether they are classified primarily as communitarian, egalitarian or individualistic cultures.

Countries with communitarian traditions based in either the family or other groupings have designed various mechanisms to ensure the interests of the

Table 1.2 Distribution by type of cultural framework

Communitarian Egalitarian Individualistic

\begin{tabular}{lll}
\hline Germany & Sweden & US \\
Netherlands & New Zealand & \\
Japan & UK
\end{tabular}

Estonia

Italy 
various communities involved in home care. In contrast, egalitarian cultures, although representing divergent political systems, all place emphasis on the entitlement to a range of home care services and on a societal commitment to provide such care on those grounds. On the other hand the US, with its rugged individualism, elevates the individual right to be left alone - for example, not to have to pay for such services through taxes - above the welfare of the community and largely sees home care as an individual or private responsibility. As such, the different cultural frameworks of home care are also highly gendered, with communitarian and individualistic frameworks relying heavily on unpaid female labour of love (by design and default, respectively) while the egalitarian framework largely treats such care as a public responsibility. Although rights and entitlements are often used synonymously, entitlements suggest a concern for equality that can be found only in the notion of positive rights. In contrast, the individualistic version of negative, selfcentred rights lacks the social dimension found in egalitarian and communitarian societies. A brief look at the most defining characteristics of each of our countries will put this in perspective.

\section{Germany}

Germany has a strong tradition of voluntarism, self-help and family support embedded in Roman Catholic social teachings and the idea of 'subsidiarity'. The 1873 social health insurance, the first of its kind, built on these traditions and incorporated them in a statutory system of social solidarity (Freeman, 2000). The organisation of social health care in Germany is marked by profound continuity and is shaped by a number of principles, among them social solidarity, freedom of choice and nearly full coverage of services (Greiner and Schulenburg, 1997). Since 1995, even long-term care needs are covered by a compulsory social insurance. In contrast to social health insurance, however, the long-term care insurance is oriented towards basic needs only. In the context of the social insurance, access to health/social care is an entitlement that individuals 'earn' by virtue of paying insurance contributions. This, together with a strong legalistic approach typical of Germany, turns access to care into the right of individuals to a defined range of services. Individuals literally have 'ownership' of these services or cash benefits. The individualised right to health care also goes hand in hand with the expectation that the social health insurance provides full coverage.

\section{The Netherlands}

Another example of a communitarian country is the Netherlands, which resembles Germany in that private initiative has been a guiding principle in the 
organisation of society. Dutch society has traditionally been organised in separate segments or 'pillars' that represent different religious and political orientations. According to the Roman Catholic notion of subsidiarity and its Protestant counterpart of sovereignty, the different segments in society are to be empowered to provide for their members (Björkman and Okma, 1997). However, the 'pillarisation' of Dutch society began to weaken in the 1960s when state intervention became more pronounced as reflected in elements of universalism (Maarse, 1997). It is also at this time that home care was included in the new (social) insurance for exceptional medical risks and provided universal access to both health and social (home) care services (Coolen and Weekers, 1998: 50).

\section{Japan}

Although for many Westerners Japan is an enigma, its home care context shares much in common with Germany and the Netherlands. At its base, Japan has a communitarian, extended-family-based culture with veneration for the elderly. Although long-term care has until recently largely rested with the family, particularly women, for formal care this has meant that today universal coverage is widely accepted socially as a given. Moreover, during the last half-century traditional Japanese values have come into conflict with the infusion of a Western, largely American, individualistic culture, thus causing significant distress particularly among the older Japanese population.

\section{Estonia}

Since its independence in 1991, Estonia has adapted a Bismarckian-oriented health care system based on the principles of equality and solidarity. The health care system has undergone fundamental reforms, including the establishment of a compulsory health insurance, the decentralisation of the national health care administration, and the development of public health care (see Saks et al., 2003). The compulsory health insurance covers almost 95 per cent of Estonian residents and 80 to 90 per cent of the health care costs of insured people. The insurance guarantees almost all residents equal financial access to medical care. The home nursing service, which is also financed by the compulsory health insurance, was established in 2002. Domestic or social services are organised by the local governments. Access criteria are based on needs, and in the case of the elderly, the family situation. The local governments pay domestic services only for childless elderly. If children or grandchildren exist, the local governments decide whether and on what terms the senior citizen can receive domestic services. 


\section{Italy}

The literature often characterises the Italian welfare state as 'conservativecorporatist' but the picture is more complex, especially in relation to home care where welfare arrangements are often 'rudimentary' (Tester, 1996). Informal care by women continues to be the norm, reflecting the strong role of the family in Italian society together with the perception of care as a family obligation (Convery and Cioni, 2001; Österle, 2001). Similar to Germany, at the formal level this expresses itself in the principle of subsidiarity. This is reflected in organisation of formal services where responsibilities are unclear and highly decentralised, public funding is patchy, provision is fragmented and the level of service provision is low (Polverini et al., 2004). In relation to health care, the principle of universalism was never fully implemented and co-payments for services remain high. The same applies to local social services and in home care where private funding solutions are fundamental. At the same time, health care as well as the local social services have seen the introduction of market mechanisms, which have produced a more mixed service provision but further complicated the boundary between the two services (Tester, 1996).

\section{Sweden}

Perhaps the clearest example of an egalitarian-type culture is Sweden, which is notable for the very early provision of medical care by the state dating back to the seventeenth century and an extensive range of social services 'from the cradle to the grave'. For instance, early on, towns and cities employed doctors to provide public health care and municipalities operated free hospitals, while in rural areas the central state paid physicians to provide basic care. State involvement in health care was consolidated in the middle of the nineteenth century with the creation of county councils which were chiefly responsible for health care. A considerable expansion of health services only occurred in the post-war period, however, paving the way for the universal health care system as we know it today. In the 1950s, social care services for the elderly were set up as a tax-based universal service organised by the local governments. The historical legacy of public involvement in health and social care is combined with the principle of equality, which is deeply embedded in Swedish society. People have a right to care regardless of income or where they live (Håkansson and Nordling, 1997). This right is part of a person's citizenship and not an individually earned entitlement as in the case of Germany and the Netherlands. 


\section{New Zealand}

Although its political system compares most closely with that of the UK and its political culture retains many features of its Commonwealth heritage, like Sweden, New Zealand has a strong tradition of egalitarianism. This was clearly reflected in the Social Security Act of 1938, which committed an open-ended provision of health care to all citizens based on need. It is also demonstrated by the strong belief in the public consultation process and the view that office holders are holders of the public trust, not above it. New Zealand's egalitarian foundation is also illustrated by the 'tall poppy' belief that people who get too successful, wealthy or powerful must be cut back to size. For example, New Zealand politicians initially embraced the New Right market-centred philosophy in the 1980s and 1990s, but the public rejected attempts to restructure the health system in ways that were seen as destroying its egalitarian foundations. The result has been a series of rather bold attempts by governments of both parties to make major changes in society, only to pull back from more extreme and unpopular tactics once the public felt threatened and demanded a return to a more egalitarian system. Home care has not been high on the agenda in New Zealand, perhaps due in part to its relatively young population.

\section{United Kingdom}

In comparison to Sweden, the UK not only has had a much shorter history of public involvement in health and social care but its approach to collective action typically has been pragmatic and empirical rather than principled (Johnson and Cullen, 2000). This pattern helps to explain why after the Second World War a universal health service was introduced in what was traditionally a liberal state regime. The National Health Service (NHS), however, did not resolve the tension between laissez-faire liberalism and collectivism and as a result a rather generous entitlement philosophy has co-existed with rationed service provision (Moran, 1999). This has also meant that home (health) care for older people has been part of the NHS by default rather than design and has always co-existed with local, meanstested social care services. The health and social care divide has thus been a salient issue in as much as formal services have de facto been heavily complemented by informal care (Lewis, 2001). The fragmentation of home care contrasts with the fact that the principle of universalism together with a centralist political system makes for public expectations that health and social services be the same (or at least comparable) across the country. 


\section{United States}

The United States is the prototype of an individualistic society. ${ }^{5}$ Although individual rights are a consideration in all Western democracies, in the US rights have been elevated to a status of supremacy over collective interests. Moreover, by rights Americans mean 'negative' rights that require minimal government intervention and discount the notion of public goods, opting instead for a system where individuals largely fend for themselves. As a result, Americans are hesitant to sacrifice perceived individual needs for the common good. Moreover, when individual rights and the common good come into conflict, individual claims take precedence. Reinforcing the predominance of negative rights, US culture is also predisposed towards progress through technological means. The result has been an unrealistic dependence on technology to fix social problems at the expense of nontechnological solutions. As a result, there has been extraordinary emphasis in the US on placing elderly people in institutional settings, particularly hospital-based ones where the best care is that which uses the most sophisticated new techniques. On the other hand, in the individualistic US informal home care support is minimal and citizens are expected to fend for themselves.

\section{Home Care Statistics}

One would expect these social and cultural variations among countries to be reflected in statistics on home care delivery and expenditures. However, one of the difficulties of analysing home care comparatively is that international statistics on home care are sparse. In part this is due to the diverse, informal and largely non-public sources of funding, which make it difficult to capture in one single figure how much is spent on home care. Also, because the organisation of home care is often highly decentralised, it is difficult to gather standardised figures across different localities let alone different countries. Not surprisingly, the statistics are often incomplete and in the main are not directly about home care. As a result, one usually can only extrapolate from trends in the more general category of long-term care or of institutionallybased care, for which more statistics are available, in order to get a sense of the degrees of de-institutionalisation in different countries. The question as to whether de-institutionalisation has been followed by the development of alternative formal home care services, however, remains unanswerable to a large degree.

The percentage of over 65 year-olds receiving formal home care services in Table 1.3 offers a more direct indication of the relative size of home care services. In the Netherlands, Sweden and the US more than 11 per cent of 
older people receive formal help at home. With 16 per cent, the US is a clear outlier. This figure, however, probably does not reflect a generosity on the part of government but rather the vigorous marketing of many private, long-term care insurance plans. In the other countries, between 9.6 per cent (in Germany) and 3 per cent (in Italy) of older people receive formal home care services. Although in all countries only a minority of older people receive formal help, the variation among countries is clear. However, this might disguise the fact that many older people living in their own home rely on informal care from female relatives. With the exception of Italy and Japan, in all countries the percentage of older people receiving formal home care is greater than the percentage living in institutions. This highlights the importance of home care for older people. It is at home where the majority of older people reside.

\section{Table 1.3 Percentage of population aged 65 and over in different care settings}

In institutions Receiving formal help at home

$\begin{array}{llr}\text { Estonia* } & & \\ \text { Germany } & 6.8 & 9.6 \\ \text { Italy } & 3.9 & 3.0 \\ \text { Japan } & 6.0 & 5.0 \\ \text { Netherlands } & 8.8 & 12.0 \\ \text { Sweden } & 8.7 & 11.2 \\ \text { UK } & 5.1 & 5.5 \\ \text { US } & 5.7 & 16.0\end{array}$

Note: $* 2.6 \%$ Tallinn (capital), $1.5 \%$ Tartu (domestic service only), taken from Saks et al. (2003).

Source: Jacobzone (1999).

Despite the inconclusive trends in the provision of institutionally-based care, the spending on other types of services for the elderly and disabled has increased significantly. Table 1.4 lists per capita public expenditure on day care, rehabilitation, home help services and other services in kind. With the exception of New Zealand and the US, in all of our countries public spending increased between 1983 and 1998, ranging from twofold or less in Italy, the Netherlands and the UK to over fourfold in Japan. Sweden continues to spend the highest per capita by far and jumped by almost fourfold during these years.

Preliminary data on expenditures for home care across these countries 
Table 1.4 Per capita public expenditure on services for the elderly and disabled (in US dollars)

\begin{tabular}{lrrrrr}
\hline & $1983^{1}$ & 1988 & 1993 & $1998^{2}$ & $\begin{array}{l}\text { Rates of } \\
\text { increase }\end{array}$ \\
\hline Germany & 44 & 62 & 111 & 166 & 3.8 \\
Italy & 21 & 33 & 44 & 42 & 2.0 \\
Japan & 17 & 24 & 44 & 73 & 4.3 \\
Netherlands & 72 & 77 & 98 & 102 & 1.4 \\
New Zealand & 4 & 4 & 4 & 1 & $\mathrm{n} / \mathrm{a}$ \\
Sweden & 202 & 289 & 571 & 791 & 3.9 \\
UK & 54 & 76 & 114 & 116 & 2.1 \\
US & 1 & 13 & 15 & 12 & $\mathrm{n} / \mathrm{a}$ \\
\hline
\end{tabular}

Notes:

1. This is the first year for which figures are available.

2. The figures refer to 1997, the most recent year for which figures are available.

Source: OECD (2001b).

demonstrate considerable variation as well. Table 1.5 shows total expenditure on home care as a percentage of GDP ranging from 0.02 per cent in Japan to 0.82 per cent in Sweden. Similar differences are apparent in the public and private distributions for home care and the comparison with spending on institutional care, which in most countries represents multiples of home care spending. Again the limits of the data must be reiterated, however, because they are unlikely to reflect the costs of informal home care supplied by family and friends. These issues will be analysed in more detail in the substantive chapters on formal and informal care givers.

The analysis of available statistics has given a preliminary overview of home care in our countries. In terms of both funding and provision, formalised home care is marginal in these countries, more so in some than in others. At the same time there is no clear evidence for systematic moves away from institutionally-based care. Nevertheless, more public money is being spent on alternative services for older people, such as day centres and home helps. Most importantly, the analysis of the statistics highlights the need to locate home care in the different health and social welfare systems in which it is embedded. While the delivery, funding and rules of home care will be examined in detail in the following chapters, it is useful here to provide a quick review of the complicated nature of governing this amorphous and inclusive area of home care. 
Table 1.5 Expenditures on long-term care as a percentage of GDP, 2000

\begin{tabular}{|c|c|c|c|c|c|c|c|}
\hline & \multicolumn{2}{|c|}{ Public expenditure } & \multicolumn{2}{|c|}{ Private expenditure } & \multicolumn{3}{|c|}{ Total expenditure } \\
\hline & Home & Institutional & Home & Institutional & Home & Institutional & Total \\
\hline Germany & 0.43 & 0.52 & 0.04 & 0.36 & 0.47 & 0.88 & 1.35 \\
\hline Japan & 0.02 & 0.69 & 0.00 & 0.07 & 0.02 & 0.76 & 0.78 \\
\hline Netherlands & 0.56 & 0.75 & 0.05 & 0.08 & 0.61 & 0.83 & 1.44 \\
\hline New Zealand & 0.11 & 0.34 & 0.01 & 0.22 & 0.12 & 0.56 & 0.68 \\
\hline Sweden & 0.78 & 1.96 & 0.04 & 0.10 & 0.82 & 2.07 & 2.89 \\
\hline UK & 0.32 & 0.58 & 0.09 & 0.38 & 0.41 & 0.96 & 1.37 \\
\hline US & 0.17 & 0.58 & 0.16 & 0.39 & 0.33 & 0.96 & 1.29 \\
\hline
\end{tabular}

Source: OECD (2004). 


\section{DEVELOPMENTS IN HOME CARE GOVERNANCE}

Thus far we have outlined what we mean by home care and home care governance and have introduced the need to analyse variation across countries. We now turn our attention to the dynamics of home care brought about by demographic, social and economic changes. Although there are many demographic factors impacting on home care, such as increased mobility and immigration, the single most important and ubiquitous is the ageing of populations. Similarly, while social changes are many, the individualisation and liberalisation of societies have led women especially to articulate their needs in different ways, which have considerable ramifications for home care. Finally, often pulling in opposite directions, economic constraints on the one hand mean that traditional home care givers are forced into the workplace to make ends meet and on the other mean that public resources have become more scarce, thus squeezing traditional welfare systems. Because of their importance for the governance of home care, we now look at each of these sets of dynamics in turn.

\section{The Problem of Ageing Populations}

There is no doubt that a major reason forcing all countries to intensify the need to address home care and to engage more actively in governing home care is the ageing of their populations. As illustrated in Table 1.6, all countries will experience significant ageing of their populations over the next 30 years. Although the ageing process is taking place earlier and more rapidly in some countries, all are expected to experience the ageing process that began in the

Table 1.6 Percentage of population aged 65 and over

\begin{tabular}{lrrrrrrr}
\hline & 1960 & 1980 & 1990 & 2000 & 2020 & 2030 & 2040 \\
\hline Estonia & - & \multicolumn{1}{c}{-} & 15.5 & - & $19-20$ & - & \\
Germany & 10.8 & 15.5 & 15.3 & 16.8 & 21.7 & 25.8 & 27.6 \\
Italy & 10.5 & 13.2 & 14.9 & 17.6 & 19.4 & 21.9 & 24.2 \\
Japan & 5.7 & 9.1 & 12.1 & 16.7 & 20.9 & 21.0 & 22.7 \\
Netherlands & 9.0 & 11.5 & 12.8 & 13.6 & 18.9 & 23.0 & 24.8 \\
New Zealand & 8.7 & 9.7 & 11.1 & 11.7 & 15.3 & 19.4 & 21.9 \\
Sweden & 11.8 & 16.3 & 17.8 & 17.8 & 20.8 & 21.7 & 22.5 \\
UK & 11.7 & 15.0 & 15.7 & 15.7 & 16.3 & 19.2 & 20.4 \\
US & 9.2 & 11.2 & 12.4 & 12.3 & 16.2 & 19.5 & 19.8 \\
\hline
\end{tabular}

Sources: OECD (1988); for Estonia: Saks et al. (2003). 
1970s and is now accelerating particularly in the non-European countries, which currently have younger populations. The primary cause of the ageing of Western societies is the precipitous decline in fertility rates, which has naturally increased the proportion of elderly. This trend is exaggerated, however, because the sharp upturn in birth rates after the Second World War produced a bloated age cohort in the 'baby boom' generation, the first wave of which is now approaching retirement age. Even if life expectancy is not lengthened over the coming decades, this upsurge of ageing baby boomers combined with low fertility rates guarantees an increasing proportion of elderly candidates for home care.

A complicating factor is increased life expectancy. In the 30 years between 1950 and 1980, life expectancy at birth on average increased by 8.5 years for females and 6.0 years for males. These sizeable gains result from improved social conditions, health habits, and the new capacities of medicine to reduce infant mortality and extend the life span. Ironically, although our advances in medical science can significantly extend the lives of the older patients, often the lives saved are at a lower quality of life and a frailty which requires even more social care over extended periods of time. According to Eurostat, these big rises in the elderly will have 'profound consequences' for social protection systems: 'EU-wide, the share of the elderly in the total population is expected to rise from $21 \%$ now to around $34 \%$ by 2050 . Those $80+$ are predicted to rise from $4 \%$ of today's population to some $10 \%$. By 2050, 37 million people are expected to be octogenarians+' (Eurostat News Release, 1999).

These ageing trends are accompanied by two changes in the population structure that are critical for home care. First, within the overall trends towards older populations is the ageing of these elderly populations themselves. At present the most rapidly growing segment of the elderly population is the cohort aged 80 and over, which currently represents the age group with the greatest need for home care. As illustrated in Table 1.7, by 2050 this proportion is expected to climb to over 30 per cent across Western countries. The second trend relates to the sex composition of the elderly population. As

Table 1.7 Estimated age structure of proportion of the population aged 65 and over, $O E C D$

\begin{tabular}{lcccccccc}
\hline & 1980 & 1990 & 2000 & 2010 & 2020 & 2030 & 2040 & 2050 \\
\hline $65-69$ & 34 & 32 & 31 & 32 & 31 & 31 & 27 & 26 \\
$70-79$ & 48 & 46 & 48 & 44 & 46 & 46 & 47 & 43 \\
$80 /$ over & 18 & 22 & 22 & 24 & 22 & 24 & 26 & 31 \\
\hline
\end{tabular}

Source: OECD (1988: 23). 
a result of their longer life expectancy, women outnumber men by significant margins in the elderly age cohorts. Furthermore, the sex imbalance increases with age, meaning that as the very elderly cohort expands, the proportion of elderly women will increase. This is particularly crucial for home care, since traditionally women have borne the brunt of home care but now also represent the largest group of users.

\section{Social Trends}

Reinforcing these demographic shifts are the substantial social transformations in modern societies that accompany the individualisation and liberalisation of traditional values. Just at the time when there is an increased need for long-term care giving, social changes have begun to undermine traditional, largely informal and family-based care, mechanisms of care for the elderly. The decline in the extended family and more recently the nuclear family, increased mobility, and trends towards secularisation have undermined traditional support systems and put added pressures on governments to fill the gap.

Moreover, in combination with the economic constraints discussed below, social movements towards equality for women have encouraged more women today to seek fulfilment through a career, with the result in many countries of reducing the supply of informal, unpaid care givers. Within this new cultural milieu, women are less likely than in the past to be willing or able to forgo paid employment to serve as a care giver. Table 1.8 shows the magnitude of the increase of women in the workplace in our countries. Although women continue to provide significant levels of long-term care for family members, coverage is saturated and as a proportion will continue to decrease just when

Table 1.8 Labour force participation rates of women, 1973-2003

\begin{tabular}{lllllll}
\hline & 1973 & 1979 & 1983 & 1993 & 1999 & 2003 \\
\hline Germany & 50.3 & 52.2 & 52.5 & 61.8 & 63.0 & 64.5 \\
Italy & 33.7 & 38.7 & 40.3 & 43.3 & 45.5 & 48.3 \\
Japan & 54.0 & 54.7 & 57.2 & 61.8 & 59.5 & 59.7 \\
Netherlands & 29.2 & 33.4 & 40.3 & 56.0 & 64.4 & 68.4 \\
New Zealand & 39.2 & 45.0 & 45.7 & 63.2 & 67.4 & 69.3 \\
Sweden & 62.6 & 72.8 & 76.6 & 75.8 & 76.0 & 76.9 \\
UK & 53.2 & 58.0 & 57.2 & 65.3 & 68.4 & 69.2 \\
US & 51.1 & 58.7 & 61.9 & 69.0 & 70.7 & 69.7 \\
\hline
\end{tabular}

Sources: 1973-93, OECD (1996); 1999-2003, OECD (2004). 
the need for home care for the elderly deepens. As a result, the demands for formal long-term care services will increase to meet expanding need, and such services will depend increasingly on public funds. Those countries without adequate planning and funding infrastructures for long-term care are especially vulnerable to severe problems in this regard.

\section{Economic Trends}

All Western countries are experiencing difficulties in meeting expanding demands for long-term care and other services as jobs are lost in globalisation, their populations age, and public resources become scarcer. Home care is seen by some observers as a way to square the circle among these demographic and cost pressures (Duff, 2001). This is based on the presumption that care in the home is much cheaper than care provided in labour-intensive hospitals and nursing homes. Substituting care in the home for institutionally-based care is also said to be better for older people, because home-based settings make it easier to combine formal with informal care arrangements.

This shift in priorities also reflects the more critical attitudes towards the institutionalisation of older people that emerged in the 1970s (Jenson and Jacobzone, 2000). Substitution policies refer to a set of policies that aim to replace institutionally-based care with care in the home and related settings. De-institutionalisation driven by either financial or humanitarian motivations, however, without sufficient development of non-institutional settings can result in additional demands on women as informal carers (ibid.). When assessing the provision of home care it is important to look at how services are organised and who provides these services. Both aspects give an indication of the degree to which home care is integrated into health and social care systems and influences the level of service provision.

\section{The Dynamics of Home Care Governance}

These three sets of factors - demographic, social and economic - have combined to create a dynamic and fluid environment for home care, especially in terms of finance. The ways in which home care is funded are significant in two respects. Security of funding, together with the relative level of (public) funding, are important factors shaping the provision of home care services. Funding arrangements also give an indication of the extent to which home care is an integral part of a health and/or social welfare system. The funding sources of formal home care can range from taxes and social insurance contributions to out-of-pocket payments and private insurance. The first two make for secure if not necessarily sufficient funding, whereas out-of-pocket payments are the least reliable and equitable sources of funding. The funding 
of home care is typically highly mixed and a substantial amount of home care is funded out of out-of-pocket payments. Moreover, public funding is often not secure and hardly sufficient and has to be supplemented by out-of-pocket payments.

Furthermore, often the level of service provision is very basic and involves a diverse range of providers. As a result, the emphasis on welfare mix competes with the policy goals of integrating services across different providers and reducing the divide between health and social care. Moreover, the discussion thus far has focused only on formal home care, that is, the care provided by paid staff with formal training/qualifications. Home care becomes even more complicated when it is realised that the majority of home care has traditionally been and still is provided by family and friends, that is, informal unpaid carers. Tester (1996), in her comparison of community care in a number of European countries and the US, estimates that informal carers provide 75 to 80 per cent of care, a figure largely borne out across many studies. With cost containment in health care and inadequate resources to develop home care services, the burden on informal carers actually has increased in recent years. Substitution policies also mean that home care services are regularly targeted at highly dependent older people who would otherwise require institutional care.

We would expect variation across countries regarding the importance of informal care in relation to underlying cultural assumptions about the role of the family (and women in particular) in care giving. In those countries where family bonds and collectivist values are traditionally strong, care-giving responsibilities of families should be more extensive with the opposite applying to countries where values of individual independence dominate. In the latter countries it will be more accepted that care giving be in the hands of paid professionals or at least that they complement informal care. With home care now on the political agenda of many countries, interest in informal care has broadened. Home is where the vast majority of older people live with their spouses and partners, and home is also one of the places where older people meet with friends and family. The spatial interface of personal relationships and home care facilitates the provision of informal care. Not surprisingly, most home care policies have acknowledged the importance of informal care. From a comparative perspective, the interesting question is what this acknowledgement means in practice and how governance defines the interface between formal and informal care.

Here a number of considerations come into play. In times of resource constraints it seems inevitable that home care systems will continue to rely on informal care. Compared to formal care this is the less costly option, but only if the costs associated with informal care (such as lost income and pension entitlements) are ignored (Jacobszone, 1999). Another consideration is that the 
expansion of formal care might lead to the reduction of the provision of informal care and even create additional demands for formal care (Jamieson, 1996). This can be either a desirable or an undesirable policy outcome, although studies indicate that substitution is not necessarily straightforward either way. For moderately disabled older people, home-based care is likely to be the best (and most efficient) option. In contrast, for severely disabled older people, institutional care is more appropriate (Jacobzone, 1999). If anything this suggests that substitution needs to be tailored to the particular circumstances of individuals (Low et al., 2000). Moreover, even in countries with relatively high levels of publicly-funded formal care, families, primarily women, continue to play a significant role as informal carers. Nevertheless, the pool of informal carers is ultimately limited and home care cannot continue to function without formal care (Twigg, 1996).

Policies on the informal aspects of home care pick and mix considerations about the interface between the formal and the informal in distinct ways. In turn they are shaped by cultural assumptions about the role of the family in care giving. At the same time, it is interesting that in recent years many countries have experimented in one way or another with a more explicit integration of informal care into the formal health system. Twigg (ibid.) has developed a typology for understanding the range of relationships that exist between welfare agencies and informal carers. She distinguishes the following types: carers as resources where carers are taken for granted; carers as co-workers where carers are treated instrumentally to ensure the continuation of their caring activities; carers as co-clients where agencies are concerned with the needs of carers in their own right; and superseded carers where agencies aim to replace carers with paid formal care staff.

Home care therefore is full of contradictions. Home care is varied and diverse yet it is an indispensable need across all countries. Home care is hardly visible in international statistics yet it is central to the health and well-being of an increasing number of people. Home care funding and provision is often fragmented, yet home care is of increasing importance on the policy agenda of many countries. These are some of the salient contradictions in home care. An interesting question is how are recent policy trends and developments altering home care in response to the demographic, social and economic pressures discussed earlier?

The provision of home care services is diverse and this diversity means a greater number and wider range of providers. This requires greater coordination but at the same time makes coordination and ultimately the integration into the health system more difficult. Problems of coordination can be exacerbated by a sharp divide between different types of care (for example, health and social care in the UK), the absence of national legislation (as in the case of the US) or highly decentralised governance structures (as in the case 
of Sweden). Also it is likely that diversity of provision does not necessarily translate into adequate levels of provision. This gives credence to the more general tenet that in times of cost containment increased diversity of provision often coincides with a targeting of services to those in greatest need or to those without any other means.

Regardless of the policy trends in the provision and funding of services, home care remains overwhelmingly informal women's work. In comparison to formal home care, informal women's work continues to be cheap and readily available in most countries. Irrespective of differences among political systems and cultural attitudes towards the role of women, however, the majority of countries have started to acknowledge the centrality of informal care. This takes different forms, ranging from the gender-blindness of liberal welfare traditions (such as in the US) and gender-specific expectations in traditionally male-dominated collectivist societies (such as in Japan), to the ideology of welfare society (such as in the UK and Sweden), the revival of subsidiarity (in Germany), and payments of carers (such as in Germany and the Netherlands). What has changed is that the marginality of informal home care has come more sharply into focus and the demographic, social and economic pressures for more far-reaching and meaningful governance of this area has intensified.

\section{CHAPTERS THAT FOLLOW}

Chapter 2 explains the foundations of the analytical framework and identifies its innovative features. It defines home care governance as consisting of a range of issues around the organisation of formal care services, the identification of informal care, the definition of the professional territories of care workers and the terms under which care users are included in home care. The chapter explores different approaches of analysing home care governance and presents the social care and the public policy literature as two complementary theoretical perspectives. It uses the questions raised by each body of literature to build a more inclusive conceptual framework for analysing and explaining home care governance across countries. Chapter 3 extends this introduction to the empirical chapters by demonstrating how to conceptualise the context of home care governance, particularly the institutions and ideas that are its key components. It thus provides a systematic analysis of the contexts of home care governance across our countries.

Chapter 4 focuses on formal care services and analyses governance arrangements as related to their organisation. Such governance arrangements are becoming higher on the political agenda in many countries, reflecting the perception that the needs of an increasingly older, and often politically astute, population are confronted with falling resources of informal care and 
constrained financial resources. Governance is therefore caught between expanding and containing formal care services, and the chapter analyses which direction governance is taking across different countries and how this can be explained. Prevailing ideas about gender relations in the labour market are particularly important here, and are also reflected in the dominant modes of funding and providing formal care services. This chapter analyses governing arrangements in relation to formal care services in terms of the relative degrees of integration and fragmentation, looking at governing through provision, funding and rules. From a cross-country comparative perspective, the chapter also explores which types of ideas and institutions as the context of home care governance provide more or less favourable conditions for relatively integrated governing arrangements.

Chapter 5 turns attention to informal home care and looks at the changing boundaries between formal and informal care. The focus is on the integration of informal care in the overall provision of care embedded in different institutional settings and its relationship to daily caring activities on a micro level, as well as the consequences for the relations between men and women, social classes and ethnic groups in society. The new approaches concern the distribution of care activities between the family or informal sector and different sectors located in the public sphere, the ascription of societal and family responsibilities and the distribution of costs. The new regulations extend mechanisms of governance to the area of informal care within the family framework and establish thus a mode of coordination which includes the state, market, associations and family. The chapter also examines employment contracts regarding wages and regulations on the labour market and the control of the caring activities.

Chapter 6 turns attention to the governance arrangements relating to care workers in home care. The formalisation of care arrangements, especially as it occurs under tight financial constraints, has raised issues about the boundaries of paid, qualified care workers in relation to informal carers and about the level of training required for different care tasks. This leads to conflicts between professional and economic interests, and this chapter examines what shape this conflict takes in different contexts and how this in turn influences governing arrangements concerned with defining professional territory across countries. Here the analysis looks at a number of factors, including prevalent ideas about gender relations and how they influence ideas about the right size and composition of professional territories in home care, the diversity of care workers as a group and how this relates to different degrees of plasticity of care, the relative existence of alliances between care workers and other actors, and the relative degree of institutional integration of funding and provision arrangements, and its importance as an incentive for developing home care as a territory for paid and qualified care workers. The discussion of the relative 
health and social care occupational orientation of care workers suggests that social care orientations dominate and that this is also closely associated with a relatively weak institutionalisation of care work as an occupational activity. The variations among countries found in Chapter 6 point to important countryspecific combinations of dissimilar aspects of the institutional context of home care governance, in particular the relative support for female employment and for formal care services, respectively.

Using the notions of consumer and citizen, Chapter 7 analyses the consequences of processes of individualisation in society by contrasting the concepts of consumerism and social and citizenship rights. As individual actors, the care recipients themselves are examined according to their opportunities to determine their individual care arrangement. The analysis looks at elements of the institutional contexts which affect the actions of the individual and collective actors such as the definition and implementation of social rights, related modes of funding via taxes or social insurance contributions and eligibility criteria, as well as features of service organisation such as diversification and offers and genuine choice. Significantly, both aspects are strongly embedded in specific mixes of different modes of coordination of home care, that is, mainly state regulations and market. The chapter finds that with the introduction of the long-term care insurance, the role of the users has been changed within a newly regulated care infrastructure oriented towards market principles.

The concluding chapter returns to the comparative context of governing home care. It attempts to categorise governing arrangements regarding the funding, delivery and rules of home care, and the formal/informal mix based on the factors identified in the substantive chapters as the most crucial. It summarises the findings concerning the logics and instruments of home care governance introduced in Chapter 1 and reviews what we have learned about patterns and differences in home care governance across these countries. This chapter also analyses to what extent these disparate countries are dealing with the challenges raised by home care. How well are the many challenges of the twenty-first century being translated into workable governance arrangements and how do the variable policies reflect the institutional and value differences across these countries? Finally, are governance initiatives across these countries leading to a policy convergence in home care in the light of the trends noted above or continued divergence?

\section{NOTES}

1. The concept of governance and its implications for the study of home care are extended in Chapters 2 and 3 , which map out the ideas and institutions that form the context of the governance of home care in our countries. 
2. This chapter presents broader more general political, cultural and historical contexts, while Chapter 3 defines these contexts in considerably more detail.

3. We have not expanded our analysis to include developing nations because we felt this would inextricably complicate the analysis. Although inclusion of a sample of such countries might be interesting, we believe that this more focused analysis is more fruitful at this stage.

4. In relation to the UK, the political devolution since the late 1990s has meant that home care policies in the four countries of the UK have started to diverge. The analysis presented in this book is primarily about policies as they apply to England. As the focus of the analysis is on comparing between (rather than within) countries, the analysis refers to the UK throughout the book.

5. The closest other being Australia. 